\title{
Internações por esquistossomose mansônica no estado da Bahia entre 2012 e 2016
}

\author{
Hospitalizations by mansonic schistosomosis \\ in the state of Bahia between 2012 and 2016
}

\section{Luciano Sá Teles de Almeida Santos ${ }^{1}$ (]) Augusto César Costa Cardoso² (1)}

\author{
1Autor para correspondência. Universidade do Estado da Bahia (Salvador). Bahia, Brasil. lucianosateles@hotmail.com \\ ${ }^{2}$ Universidade do Estado da Bahia (Salvador), Escola Bahiana de Medicina e Saúde Pública (Salvador). Bahia, Brasil. acccardoso@uneb.br
}

\begin{abstract}
RESUMO | OBJETIVO: descrever a situação epidemiológica das internações por esquistossomose mansônica no estado da Bahia entre 2012 e 2016. MATERIAL E MÉTODOS: estudo epidemiológico e descritivo onde foram registrados o número e o perfil das internações por esquistossomose mansônica no estado da Bahia no período entre 2012 e 2016. Os dados foram obtidos na base de dados DATASUS do Ministério da Saúde. As variáveis utilizadas foram as que já existiam no sistema referentes à esquistossomose, com algumas adaptações: sexo, faixa etária, cor/etnia, número de internações, valor total dos serviços e macrorregiões de saúde. RESULTADOS: no período analisado foram registradas 109 internações, sendo a maior parte população do sexo masculino (67\%) e na faixa etária entre 15 a 59 anos (61\%). A distribuição das internações por áreas geográficas foi heterogênea, sendo a macrorregião Leste da Bahia com maior frequência (36) e maiores gastos com as internações ( $R \$ 14967,28)$. CONCLUSÃO: a esquistossomose ainda se mantém como um problema de saúde pública no estado da Bahia gerando custos para o sistema de saúde e interferindo negativamente na qualidade de vida e na produtividade dos indivíduos.
\end{abstract}

DESCRITORES: Esquistossomose mansoni. Epidemiologia. Hospitalização.

\begin{abstract}
OBJECTIVE: to describe the epidemiological situation of hospitalizations for schistosomiasis mansoni in the state of Bahia in the period between 2012 and 2016. MATERIAL AND METHODS: epidemiological and descriptive study where the number and profile of hospitalizations for schistosomiasis mansoni in the state of Bahia in the period between 2012 and 2016 were recorded. Data were obtained from the DATASUS database of the Ministry of Health. The variables used were those that already existed in the system related to schistosomiasis, with some adaptations: gender, age, color / ethnicity, number of hospitalizations, total value of services and macro-regions of health. RESULTS: in the analyzed period, 109 hospitalizations were recorded, with the majority of the population being male (67\%) and in the age group between 15 to 59 years (61\%). The distribution of hospitalizations by geographic areas was heterogeneous, with an Eastern macro-region of Bahia being more frequent (36) and higher spending on hospitalizations ( $\$$ 14967.28). CONCLUSION: schistosomiasis still remains a public health problem in the state of Bahia generating costs for the health system and negatively interfering with the quality of life and productivity of individuals.
\end{abstract}

DESCRIPTORS: Schistosomiasis mansoni. Epidemiology. Hospitalization. 


\section{Introdução}

A esquistossomose mansônica é uma doença infecciosa parasitária que pode ter apresentação aguda ou crônica causada pelo trematódeo digenético Schistosoma Mansoni, sendo o homem o principal reservatório e caramujos do gênero Biomphalaria como hospedeiros intermediários. É uma condição que tem uma significativa prevalência mundial, sendo que sua transmissão já foi reportada em mais de 78 países da América Latina, África e Ásia. Estima-se que nesses países existam cerca de 200 milhões de indivíduos infectados ${ }^{1-3}$.

O Brasil é país com maior prevalência de esquistossomose na América Latina, sendo essa doença considerada um problema de saúde pública, acometendo principalmente crianças e adultos jovens em sua fase produtiva de vida ${ }^{4}$. Estima-se que no Brasil, cerca de 43 milhões de pessoas estão exposta ao risco de infecção e que mais de 6 milhões de pessoas estão infectadas ${ }^{1,5}$.

A área endêmica se estende do Maranhão até Minas Gerais, englobando 19 estados. A região Nordeste é a área com maior endemicidade, sendo que Alagoas, Sergipe, Bahia e Pernambuco são os estados com maior prevalência da doença ${ }^{6}$. No ano de 2006, na Bahia, esta doença foi notificada em $65 \%$ dos municípios (271/417), com média de 165,8 internações/ano, 40,2 óbitos/ano e prevalência de $5,4 \% 2$.

Nas localidades endêmicas, os dois mais importantes hospedeiros intermediários são os caramujos de água doce Biomphalaria glabrata e Biomphalaria straminea da família Planorbidae. O habitat natural destas espécies consiste de corpos de água com pouca corrente ou água estagnada, preferencialmente em áreas rasas de rios, riachos, canais e planícies de inundação ${ }^{3}$.

Clinicamente, a doença pode ser assintomática ou se apresentar em duas fases. Na fase inicial (1) pode ocorrer a dermatite cercariana causada pela penetração da cercária na pele do indivíduo. Além disso, os sintomas agudos da esquistossomose podem aparecer entre 3 a 7 semanas após a exposição e são caracterizados por tosse, febre, anorexia, dor abdominal e cefaleia. Cerca de 40 a 60 dias após a infecção inicial, ocorre a fase crônica, que é caracterizada pela formação dos ovos do esquistossômulo. As manifestações clínicas dessa fase variam de acordo com a carga parasitária e a resposta imune do hospedeiro. Os pacientes podem cursar com as formas hepatointestinal, hepatoesplênica e mielorradiculopatia esquistossomótica, além de poder ocorrer em outros tecidos?

Inicialmente, a ação imunológica consiste em uma resposta celular aguda de predomínio direcionada à forma adulta do invasor. Em seguida, quando ocorre a deposição dos ovos do Schistosoma mansoni nos tecidos do hospedeiro, a resposta é gradualmente modificada para o tipo Th2, cursando com lesões decorrente da ação inflamatória granulomatosa composta por macrófagos englobando o miracídio e com muitos eosinófilos e linfócitos em torno?

A história clínica e epidemiológica, assim como o exame físico, pode orientar o diagnóstico. No entanto, como a esquistossomose em suas diversas formas clínicas se assemelha a muitas outras doenças, o diagnóstico de certeza só é feito por meio de exames laboratoriais. Os métodos diagnósticos laboratoriais podem ser diretos e indiretos 7,9 .

No método direto investiga-se a presença de ovos do S. mansoni nas fezes ou nos tecidos ou antígenos circulantes do parasita. A análise das fezes utilizando a técnica de Kato-Katz é a mais utilizada pelos programas de controle, recomendada pela Organização Mundial de Saúde (OMS) e permite avaliar a intensidade da infecção. Os métodos indiretos são baseados na reação de antígeno anticorpo e que têm aplicação quase sempre em inquéritos epidemiológicos e em casos de diagnósticos difíceis pelos métodos diretos. Atualmente, os seguintes métodos indiretos são utilizados para diagnóstico da esquistossomose: ensaio imuno enzimático (ELISA), imunofluorescência (IF), reação peri-ovular (RPOV)?․ 
Outros exames também podem ser importantes para investigação e manejo da esquistossomose. Os exames de imagem como radiografia, ultrassonografia, endoscopia digestiva e ressonância nuclear magnética podem ser utilizados para avaliação do comprometimento orgânico decorrente da infecção por S. mansoni em suas várias formas de evolução e apresentação9-10.

O tratamento deve ser direcionado à fase de evolução da doença e varia desde o uso de medicamentos até os procedimentos cirúrgicos. Objetivando reduzir a reação inflamatória pode-se utilizar corticosteróides, porém o tratamento específico é feito com anti-parasitários (prazinquantel e oxaminiquina) que podem cursar com a cura e apresentam taxas insignificantes de resistência. As intervenções cirúrgicas são indicadas para o manejo das complicações decorrentes da forma crônica da doença, particularmente àquelas relacionadas com hipertensão portal9-10.

O controle da esquistossomose é complexo por necessitar de ações relacionadas com mudanças das condições de vida das populações expostas, de medidas de cuidado ambiental e de educação em saúde. Para que isso ocorra é imprescindível sensibilidade por parte dos gestores públicos e dos profissionais de saúde.

Por ser uma condição que tem impacto significativo sobre a vida dos indivíduos infectados assim como para o sistema de saúde, o conhecimento dos aspectos epidemiológicos atuais pode auxiliar os profissionais de saúde no manejo dos pacientes, além de contribuir na elaboração de estratégias eficazes para o controle dessa parasitose pelos gestores de saúde. Dessa forma, o objetivo deste artigo é descrever o perfil epidemiológico das internações por esquistossomose mansônica no estado da Bahia no período de 2012 a 2016.

\section{Material e métodos}

Trata-se de um estudo epidemiológico descritivo e retrospectivo. Os dados obtidos são referentes ao estado da Bahia no período de janeiro de 2012 até dezembro de 2016 disponíveis na seção Morbidade
Hospitalar do SUS (SIH) da base de dados DATASUS, tendo a coleta ocorrida nos meses de outubro e novembro de 2017.

Foram consideradas as informações referentes às internações, valores dos serviços e mortalidade devido a esquistossomose mansônica durante o período determinado. O período foi delimitado até 2016 porque era o ano mais atual em que os dados estavam completos até o momento da pesquisa.

As variáveis utilizadas foram as que já existiam no sistema referente à esquistossomose, com algumas adaptações: sexo, faixa etária, cor/etnia, número de internações, valor total dos serviços e macrorregiões de saúde.

O conjunto de dados para análise foi selecionado e obtido por meio do aplicativo TABNET a partir de suas caixas de opções (linha, coluna e conteúdo). Os dados foram transferidos para o software Excel do pacote Microsoft Office $2010 \AA$ e analisados por meio da frequência absoluta, média, além de indicadores de porcentagens e taxas. Estes são apresentados em forma de gráficos e tabelas.

Por se tratar de dados secundários de um banco de domínio público, não foi necessário submeter o trabalho ao Comitê de Ética em Pesquisa em Seres Humanos (CEP).

\section{Resultados}

Os dados que caracterizam a população de pacientes internados por esquistossomose no estado da Bahia, no período determinado estão disponíveis na Tabela 1. Vale destacar que no período analisado foram registradas 109 internações (104 em caráter de urgência e 5 eletivas), sendo que a maior frequência foi observada no sexo masculino, na faixa etária de 15 a 59 anos e quase metade dos indivíduos internados não tinha informações sobre cor/raça registradas. No período analisado ocorreram 3 óbitos entre os internados, apresentando uma letalidade de 3,72\%. 
Tabela 1. Caracterização dos pacientes internados por esquistossomose no estado da Bahia entre os anos de 2012 e 2016

\begin{tabular}{|c|c|c|c|c|c|c|}
\hline \multirow{2}{*}{ Variáveis } & \multicolumn{6}{|c|}{ Ano } \\
\hline & $\begin{array}{l}2012 \\
\text { n (\%) }\end{array}$ & $\begin{array}{l}2013 \\
\text { n (\%) }\end{array}$ & $\begin{array}{l}2014 \\
\mathrm{n}(\%)\end{array}$ & $\begin{array}{l}2015 \\
\mathrm{n}(\%)\end{array}$ & $\begin{array}{l}2016 \\
\text { n (\%) }\end{array}$ & $\begin{array}{l}\text { Total } \\
\text { N (\%) }\end{array}$ \\
\hline \multicolumn{7}{|l|}{ Sexo } \\
\hline Masculino & $15(57,7)$ & $17(65,4)$ & $7(58,3)$ & $13(59,1)$ & $15(65,2)$ & $67(61,5)$ \\
\hline Feminino & $11(42,3)$ & $9(34,6)$ & $5(41,7)$ & $9(40,9)$ & $8(34,8)$ & $42(38,5)$ \\
\hline \multicolumn{7}{|l|}{ Faixa Etária } \\
\hline Menor 1 ano & $0(0,0)$ & $1(3,8)$ & $0(0,0)$ & $0(0,0)$ & $0(0,0)$ & $1(0,9)$ \\
\hline 1 a 14 anos & $5(19,2)$ & $6(23,1)$ & $2(16,7)$ & $4(18,2)$ & $5(21,7)$ & $22(20,1)$ \\
\hline 15 a 59 anos & $14(53,8)$ & $13(50)$ & $7(58,3)$ & $13(59,1)$ & $14(60,9)$ & $61(56,0)$ \\
\hline Maior que 60 anos & $7(27)$ & $6(23,1)$ & $3(25,0)$ & $5(22,7)$ & $4(17,4)$ & $25(23,0)$ \\
\hline \multicolumn{7}{|l|}{ Cor/raça } \\
\hline Branca & $3(11,5)$ & $3(11,5)$ & $0(0,0)$ & $0(0,0)$ & $1(4,3)$ & $7(6,4)$ \\
\hline Preta & $1(3,8)$ & $2(7,7)$ & $1(8,3)$ & $0(0,0)$ & $0(0,0)$ & $4(3,6)$ \\
\hline Parda & $7(27)$ & $10(38,5)$ & $8(66,7)$ & $12(54,5)$ & $9(39,2)$ & $46(42,3)$ \\
\hline Amarela & $0(0,0)$ & $0(0,0)$ & $0(0,0)$ & $1(4,5)$ & $1(4,3)$ & $2(1,9)$ \\
\hline Sem informação & $15(57,7)$ & $11(42,3)$ & $3(25,0)$ & $9(41,0)$ & $12(52,2)$ & $50(45,8)$ \\
\hline TOTAL & 26 & 26 & 12 & 22 & 23 & 109 \\
\hline
\end{tabular}

Fonte: Ministério da Saúde - Sistema de Informações Hospitalares do SUS (SIH/SUS).

O gráfico 1 descreve a frequência das internações no período, segundo às macrorregiões de saúde do estado da Bahia. O maior número de casos foi registrado na macrorregião leste que engloba a cidade de Salvador, capital do estado. Em seguida, a macrorregião Sudoeste onde se localiza Vitória da Conquista, terceira cidade mais populosa do estado.

Gráfico 1. Número de internações por esquistossomose no estado da Bahia por macrorregiões de saúde entre os anos de 2012 e 2016

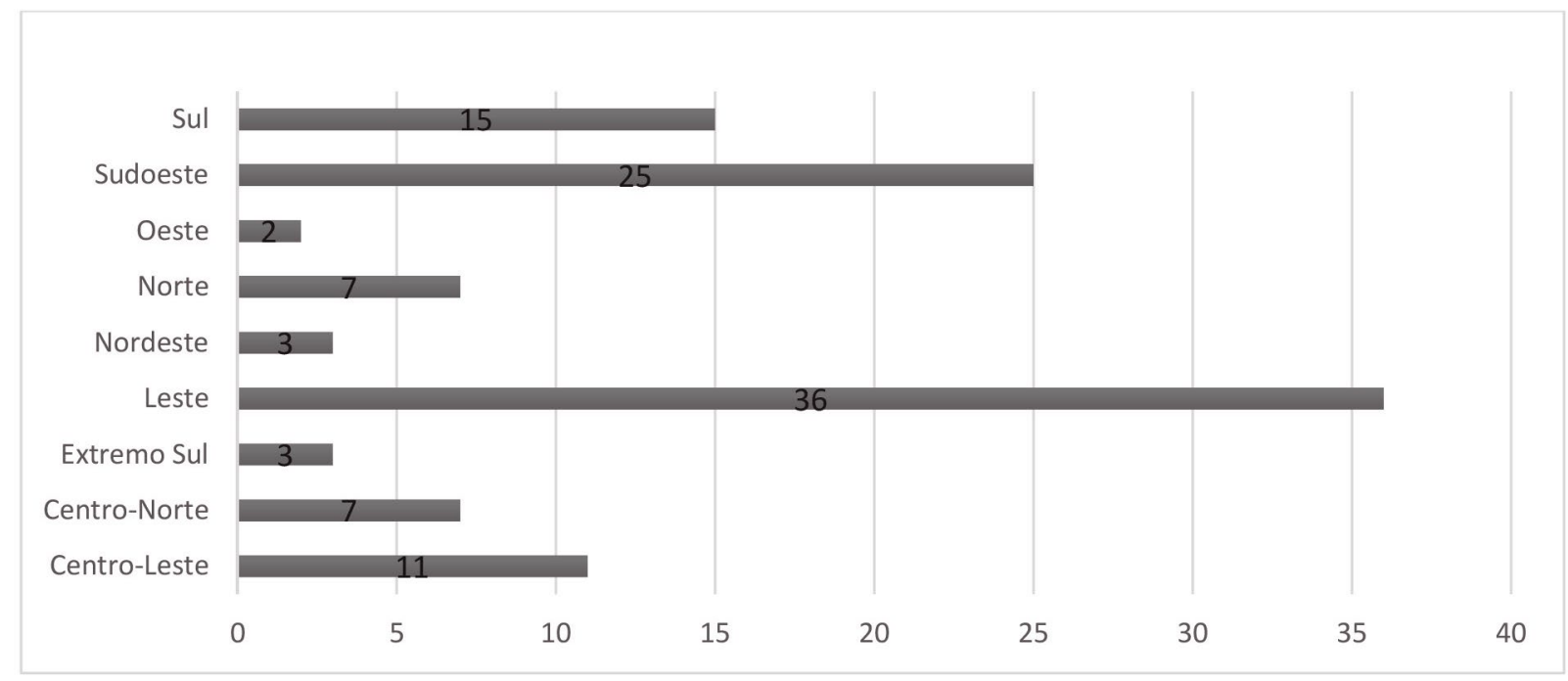

Fonte: Ministério da Saúde - Sistema de Informações Hospitalares do SUS (SIH/SUS). 
Na Tabela 2 estão disponíveis as informações sobre os valores gastos com os atendimentos durante o intervalo de tempo analisado. A macrorregião Leste foi que teve maior dispêndio financeiro. Logo na sequência vem a macrorregião Sudoeste. A macrorregião que teve o menor gasto com a doença foi a Nordeste.

Tabela 2. Valores de gastos, em reais $(R \$)$, com internações por esquistossomose por macrorregião de saúde e ano, no estado da Bahia no período de 2012 a 2016

\begin{tabular}{|c|c|c|c|c|c|c|}
\hline \multirow{2}{*}{ Macrorregião de Saúde } & \multicolumn{6}{|c|}{ Ano } \\
\hline & 2012 & 2013 & 2014 & 2015 & 2016 & Total \\
\hline Centro-Leste & 594,18 & 375,13 & 0 & 1845,38 & 593,01 & 3407,7 \\
\hline Centro-Norte & 629,23 & 0 & 0 & 189,67 & 230,05 & 1048,95 \\
\hline Extremo Sul & 221,67 & 189,67 & 0 & 189,67 & 0 & 601,01 \\
\hline Leste & 2163,09 & 5235,14 & 1029,1 & 3546,12 & 2993,83 & 14967,28 \\
\hline Nordeste & 347,7 & 0 & 189,67 & 259,62 & 0 & 796,99 \\
\hline Norte & 0 & 254,61 & 865,77 & 0 & 669,3 & 1789,68 \\
\hline Oeste & 213,67 & 0 & 0 & 735,82 & 0 & 949,49 \\
\hline Sudoeste & 1386,3 & 1244,62 & 1128,47 & 1610,28 & 1264,7 & 6634,37 \\
\hline Sul & 860,63 & 1301,92 & 189,67 & 542,83 & 417,29 & 3312,34 \\
\hline Total & 6416,47 & 8601,09 & 3402,68 & 8919,39 & 6168,18 & 33507,81 \\
\hline
\end{tabular}

Fonte: Ministério da Saúde - Sistema de Informações Hospitalares do SUS (SIH/SUS).

\section{Discussão}

Os dados encontrados nesse estudo mostram que a esquistossomose continua sendo uma condição presente no estado da Bahia. Apesar disso, o número de óbitos pela esquistossomose é relativamente baixo (3 óbitos) apresentando uma taxa de letalidade de 3,72\% entre os indivíduos internados no período.

Infelizmente, o sistema de dados não oferece maiores informações para uma melhor caracterização da amostra, assim como para determinar a forma de apresentação da doença. Outra limitação da plataforma do DATASUS é que os números podem ser subestimados já que a notificação desse agravo não é compulsória nas áreas endêmicas, de acordo com a Portaria n 1.271 de 06 de junho de 2014, da Secretaria de Vigilância em Saúde, do Ministério da Saúde. No entanto, para registro de dados operacionais dos inquéritos coproscópicos, epidemiológicos e malacológicos é utilizado o Sistema de Informação do Programa de Vigilância e Controle da Esquistossomose - SISPCE .

O Programa de Controle da Esquistossomose (PCE) surgiu na década de 1980, substituindo o antigo Programa Especial de Controle da Esquistossomose (Pece) implantado pela Superintendência de Campanhas em Saúde Pública (Sucam) na década anterior. O PCE consiste em uma série de ações como inquéritos epidemiológicos, educação em saúde e busca ativa, que objetivam reduzir o número de pessoas infectadas reduzindo assim a transmissão. Após a implantação do PCE houve uma mudança no perfil da doença em âmbito nacional, sendo reduzido o número de óbitos e de formas graves ${ }^{9,11}$.

O estado da Bahia é constituído por 417 municípios. Na plataforma do DATASUS, esses municípios estão distribuídos em nove macrorregiões de saúde: centro-leste, centro-norte, extremo sul, leste, nordeste, norte, oeste, sudoeste e sul. A regionalização da saúde faz parte de um dos princípios estruturante do Sistema único de Saúde - SUS, sendo que essa configuração atual em nove macrorregiões foi determinada em 2007 pelo Plano Diretor de Regionalização ${ }^{12}$. 
Na macrorregião leste está localizada a sede da capital do estado, Salvador, sendo essa a macrorregião mais populosa e a que apresenta maior concentração de serviços em saúde. Desse modo, muitos usuários do SUS convergem das demais regiões do estado para a macrorregião leste em busca de procedimentos especializados, além de apoio diagnóstico e terapêutico ${ }^{12}$. Em um estudo realizado por Cardim e colaboradores, 2011, foram feitas análise diagnósticas (exame coproparasitológico pelo método Kato-Katz) para essa doença na população da cidade de Lauro de Freitas, que compõe a região metropolitana de Salvador, e foram confirmados 1006 casos no período de 2006 a $2008^{3}$.

Em consequência ao maior número de internações, a macrorregião leste foi a que apresentou as maiores cifras de gastos com as internações em todos os anos, exceto em 2014, quando a macrorregião sudoeste a superou. Esses valores não refletem o custo real da esquistossomose para o sistema de saúde, já que estes se relacionam somente com os gastos durante as internações, mas há outros custos indiretos não mencionados. No Brasil ainda há uma escassez de estudos que estimem os custos da esquistossomose para o SUS.

A macrorregião centro-leste, onde está localizado o munícipio de Feira de Santana, o segundo mais populoso do estado, apresentou 11 casos no período de análise desse estudo. Em um estudo onde se analisou o número de casos de esquistossomose mansônica nesse munícipio entre 2003 e 2006, Neres e colaboradores, 2011, encontraram um total de 4205 casos. Esses autores consideraram Feira de Santana como uma área de baixa endemicidade já que apresentou prevalência inferior a $1 \%$ para essa moléstia ${ }^{13}$.

Com o processo de êxodo rural e migração, o perfil epidemiológico de algumas doenças sofreu algumas alterações. A esquistossomose, por exemplo, antes considerada uma condição rural passou a ser registrada nas cidades e grandes metrópoles ${ }^{14}$. Porém nesse estudo não foi possível determinar a origem dos casos, da zona rural ou urbana, já que só pela distribuição geográfica das internações não dá para se inferir de onde vieram esses pacientes.

\section{Conclusão}

No período analisado, foi encontrado um número notável de internações por esquistossomose mansônica no estado da Bahia. O maior número de internações foi a da população com idade economicamente ativa e do sexo masculino. A distribuição espacial dessas internações foi heterogênea. Esse fato pode estar associado à dinâmica da interação dos diversos fatores relacionados à transmissão e o controle da doença no território baiano.

A esquistossomose ainda se mantém como um problema de saúde pública gerando custos para o sistema de saúde e interferindo negativamente na qualidade de vida e na produtividade dos indivíduos. Ainda se necessita a realização de mais estudos para analisar situação na prática clínica e o impacto da doença no estado.

\section{Contribuições dos autores}

Santos LSTA participou da concepção, delineamento, busca e análise estatística dos dados da pesquisa, interpretação dos resultados e redação do artigo científico. Cardoso ACC participou do delineamento do estudo, da coleta de dados da pesquisa, interpretação dos dados e redação do artigo.

\section{Conflitos de interesses}

Nenhum conflito financeiro, legal ou político envolvendo terceiros (governo, empresas e fundações privadas, etc.) foi declarado para nenhum aspecto do trabalho submetido (incluindo, mas não se limitando a subvenções e financiamentos, participação em conselho consultivo, desenho de estudo, preparação de manuscrito, análise estatística, etc.).

\section{Referências}

1. Santos AD, Lima ACR, Santos MB, Alves JAB, Góes MAO, Nunes MAP et al. Spatial analysis for the identification of risk areas for schistosomiasis mansoni in the State of Sergipe, Brazil, 2005-2014. Rev Soc Bras Med Trop. 2016;49(5):608-615. doi: 10.1590/00378682-0137-2016 
2. Cardim LL, Ferraudo AS, Pacheco STA, Reis RB, Silva MMN, Carneiro DDMT et al. Análises espaciais na identificação das áreas de risco para a esquistossomose mansônica no Município de Lauro de Freitas, Bahia, Brasil. Cad Saúde Pública. 2011;27(5):899908. doi: $10.1590 /$ S0102-311X2011000500008

3. Leal Neto OB, Gomes ECS, Oliveira Júnior FJM, Andrade R, Reis $\mathrm{DL}$, Souza-Santos $\mathrm{R}$ et al. Biological and environmental factors associated with risk of schistosomiasis mansoni transmission in Porto de Galinhas, Pernambuco State, Brazil. Cad Saúde Pública. 2013;29(2):357-367. doi: 10.1590/S0102-311X2013000200022

4. Pereira LF, Gazzaneo AL, Melo RMPA, Tenório HC, Oliveira DS, Alves MSC et al. Clinical and laboratory evaluation of schistosomiasis mansoni patients in Brazilian endemic areas. Mem Inst Oswaldo Cruz. 2010;105(4):449-453. doi: 10.1590/S0074$\underline{02762010000400016}$

5. Martins DS, Xavier MF, Masiero FS, Cordeiro J, Thyssen PJ. Schistosomiasis in Southern Brazil 17 years after the confirmation of the fi rst autochthonous case. Rev Soc Bras Med Trop. 2015; 48(3):354-357. doi: 10.1590/0037-8682-0240-2014

6. Amaral RS, Porto MAS. Evolução e situação atual do controle da esquistossomose no Brasil. Rev Soc Bras Med Trop. 1994 (supl. III); 27:73-90.

7. Grenfel RFQ, Martins W, Drummond SC, Antunes CMF, Voieta I, Otoni A et al. Acute schistosomiasis diagnosis: a new tool for the diagnosis of schistosomiasis in a group of travelers recently infected in a new focus of Schistosoma mansoni. Rev Soc Bras Med Trop. 2013;46(2):208-213. doi: 10.1590/0037-8682-0064-2012
8. Oliveira SM, Bezerra FSM, Carneiro TR, Pinheiro MCC, Queiroz JAN. Association between allergic responses and Schistosoma mansoni infection in residents in a low-endemic setting in Brazil. Rev Soc Bras Med Trop. 2014;47(6):770-774. doi: 10.1590/00378682-0249-2014

9. Brasil. Ministério da Saúde. Secretaria de Vigilância em Saúde. Departamento de Vigilância Epidemiológica. Vigilância da Esquistossomose Mansoni: diretrizes técnicas. 4.ed. Brasília: Ministério da Saúde; 2014.

10. Vitorino RR, Souza FPC, Costa AP, Faria Júnior FC, Santana LA, Gomes AP. Esquistossomose mansônica: diagnóstico, tratamento, epidemiologia, profilaxia e controle. Rev Bras Clin Med. 2012; 10(1):39-45.

11. Silva PCV, Domingues ALC. Aspectos epidemiológicos da esquistossomose hepatoesplênica no estado de Pernambuco, Brasil. Epidemiol Serv Saude. 2011;20(3):327-336. doi: 10.5123/ S1679-49742011000300007

12. Santos AM, Assis MMA. Processo de regionalização da saúde na Bahia: aspectos políticos-institucionais e modelagem dos territórios sanitários. G\&DR. 2017;13(2):400-422.

13. Neres RCB, Araújo EM, Rocha WJFS, Lacerda RS. Caracterização epidemiológica dos casos de esquistossomose no município de Feira de Santana, Bahia - 2003 - 2006. Rev Baiana Saúde Pública. 2011;35(Supl. 1):28-37. doi: 10.22278/2318-2660.2011.v35.n0.a145

14. Enk MJ, Amaral GL, Silva MFC, Lemos DS, Carvalho AT, MartinsFilho $A O$ et al. Rural tourism: a risk factor for schistosomiasis transmission in Brazil. Mem Inst Oswaldo Cruz. 2010;105(4):537540. doi: $\underline{10.1590 / \text { S0074-02762010000400032 }}$ 